\title{
Developing a knowledge-based system for the design of innovative product creation
} processes for network enterprises

\author{
Yury F. Telnov ${ }^{\mathrm{a}}$ (D) \\ E-mail: Telnov.YUF@rea.ru
}

Vasiliy A. Kazakov ${ }^{\mathrm{a}}$

E-mail: Kazakov.VA@rea.ru

\section{Vasiliy M. Trembach ${ }^{\mathrm{b}}$}

E-mail: trembach@yandex.ru

\author{
a Plekhanov Russian University of Economics \\ Address: 36, Stremyanny Lane, Moscow 117997, Russia \\ ${ }^{\mathrm{b}}$ Moscow Aviation Institute (National Research University) \\ Address: 4, Volokolamskoe Shosse, Moscow 125993, Russia
}

\begin{abstract}
The relevance of developing knowledge-based systems used to support innovative processes for creating products and services is related to the objective need to reduce the life cycle of products under the influence of modern digital technologies in developing network enterprises. Well-known research results in the field of model-oriented design of products, processes, systems and enterprises do not fully provide semantic interoperability in the interaction of stakeholders in the innovation process. The aim of this work is to build a knowledge-based system architecture that implements semantic interoperability of network enterprise participants at various stages of the product lifecycle. The work is based on the use of a model-oriented approach to building a digital thread at all stages of the product lifecycle, an ontological approach to semantic modeling of a distributed knowledge base and a multiagent approach to organizing interaction between interested participants in the innovation process. The paper proposes a functional architecture of a knowledge-based system that includes modules for planning the innovation process, forming product value characteristics and functional requirements, construction and value chain design. A multi-level system of ontologies of the innovation process is also developed and its application in the work of functional modules that provide access to
\end{abstract}


associated knowledge bases is described. The development of knowledge-based systems based on the results obtained will allow us to find the best design solutions for the configuration of products and corresponding value chains due to the possible iteration of the innovation process and increasing the semantic interoperability of network enterprise stakeholders.

Key words: innovative process; digital thread; model-oriented approach; knowledge-based system; ontology of innovative process; stakeholders of network enterprise; value chain; multi-agent interaction; semantic interoperability.

Citation: Telnov Yu.F., Kazakov V.A., Trembach V.M. (2020) Developing a knowledge-based system for the design of innovative product creation processes for network enterprises. Business Informatics, vol. 14 , no 3, pp. 35-53. DOI: 10.17323/2587-814X.2020.3.35.53

\section{Introduction}

I nnovative processes for creating new products are characterized by a high degree of uncertainty and dynamic changes in the requirements for the products, especially at the initial stages of the life cycle associated with the definition of the product concept and subsequent design. At the same time, the trend of generally reducing the product life cycle in the conditions of widespread use of digital technologies, in particular, the technology of "digital twins" and virtualization of interaction between participants in the innovation process of a network enterprise, determines the need to develop new methods for modeling the information space at all stages of the life cycle.

The main problem of existing methods of modeling the information space in the form of project repositories [1] is the weak semantic connectivity of the information resources used, reflecting various aspects of the innovative process of creating products. As a result of emerging semantic problems, it becomes very difficult to obtain consistent design solutions formed by heterogeneous network enterprise stakeholders. This makes it relevant to develop a knowledge-based system that would ensure an integrated, consistent and dynamic use of knowledge throughout the innovation process - from the origination of the product idea to its actual implementation in an industrially manufactured product.

The solution of the problem of integrated representation of knowledge about a product and related business processes is currently based on a model-oriented approach to building systems and enterprises [2,3], the implementation of the concept of digital twins [4], as well as the concept of dynamic maintenance of product models at all stages of the lifecycle - the concept of a digital thread $[5,6]$. Semantic integrity and consistency of information sources used (semantic interoperability) is studied in works related to the construction of ontologies of enterprises [7, 8]. At the same time, the complex linking of these concepts within the framework of creating a unified system based on knowledge is far from a theoretical solution and practical implementation, especially in terms of creating mechanisms for the transition between models at different stages of the product lifecycle and interaction of stakeholders in the innovation process.

In connection with the above, the aim of this paper is to define the architecture of a knowledge-based system based on a multilevel system of ontologies of the innovation process which would ensure semantic consistency of product and process models at various stages of the life cycle, ensuring effective 
dynamic interaction of real and potential participants of a network enterprise using multiagent technologies.

\section{Methodological frameworks of knowledge-based systems creating the design of innovative processes}

The design of innovative processes for creating products of a networked enterprise, as a rule, has a nonlinear iterative nature, involving a long time of searching and testing conceptual ideas, resources for their implementation, coordination with prospective participants of the network enterprise plans to manufacture and market products and services. This dynamic nature of product design processes necessitates the use of various methods for modeling products and services, as well as related processes at all stages of the life cycle.

Theoretically, the process of creating products based on system modeling is reflected in the OMG Model Driven System Design (MDSD) approach [2]. According to this approach, any product can have a complex structure of subordinate components, which, on the one hand, must have clearly defined requirements at each time, and on the other hand, these components must be represented by a set of models in order to develop the most appropriate solutions that meet the formulated requirements. Making design solutions depends on many factors and, above all, on the availability of resources, capabilities and risks of their implementation. The assessment of capabilities and risks can be characterized by incomplete and unreliable information, as well as variability in the state of resources over time under the influence of various external conditions. These circumstances make it necessary to save various design solutions in the project repository and continuously update information about the status of work on the project.
In this regard, we should pay attention to the organization of information storage in intelligent project repositories that integrate a variety of information sources about the project using ontological tools [1]. This creates a unified information space for stakeholders in the process of creating products that belong to different categories. However, the text form that underlies the documentary organization of project information has a number of limitations related to the isolation of documents from each other. In this case, the model representation of project information provides greater connectivity of the analyzed objects and their parameters, as well as the ability to use a variety of tools for quantitative and qualitative assessment of design solutions.

The development of the concept of dynamic maintenance of information about the state of project decisions regarding the product being developed at various stages of the product life cycle is a concept that is characterized by "the use of integrated information stores, which include, in addition to collecting and analyzing primary data, tools for object representation of the domain in the form of collections of digital twins (DTC) with a description of the structure, composition of objects, their properties, as well as known relationships between them. Promising technologies for organizing integrated information storage should also include methods for their systematic description (construction) and logical and analytical processing" [4].

As a rule, a digital twin is a software and hardware complex that displays the state of components of a real product or equipment in time. The digital twin receives information from its physical counterpart, for example, using the Internet of Things (IoT) and performs, if necessary, control actions to bring the physical object to the required state. At the same time, the digital twin can perform analytical and predictive functions, analyzing large amounts of data and functioning, 
in fact, as a software agent endowed with the intellectual ability to model situations. In principle, digital twins can be combined into multi-agent systems to simulate more complex technological processes.

Digital twins are mainly used at the product operation stage, accumulating large amounts of data about the product structure or production process. This data can also be used to improve or redesign the product structure. The result of product design can be immediately reflected in the information structure of the digital twin. At the same time, the concept of digital twins is not suitable for implementing the actual product design processes. This is due to the fact that design requires not only information about the functioning of existing products, but also external information about scientific and technical developments, the behavior of competitors, suppliers, manufacturers and consumers of products.

In this regard, the development of the digital thread concept is of great interest. It considers the creation, implementation and operation of products at all stages of the life cycle [5], modeling, in fact, not individual products and processes, but interrelated processes of the enterprise and their interaction with external stakeholders in production activities. As a result, Model-Based Enterprises are formed [3], which best meets the requirements for creating flexible and dynamically formed network enterprises [9].

The Digital Thread for Smart Manufacturing Systems project includes methods and protocols that extend and complement the end-to-end flow of information that passes through the processes of product design, production, and support, enabling the integration of intelligent manufacturing systems. As a result, the life cycle of bringing the product to the production stage is shortened, while saving costs [6]. The project focuses on establishing the relationship between the product model and the production process model, which makes it possible to make adjustments to design solutions through feedback from the implementation of the production process to the product design. At the same time, the basic principles of system engineering are used in terms of requirements management, verification and validation in a clearly formalized form. However, the problems of transmitting unambiguous semantics of requirements and feedback on their implementation have not been fully resolved. In this regard, the development of the Quality Information Framework (QIF) to formalize the requirements for information exchange in terms of quality measurement is of particular interest ${ }^{1}$.

To attract external stakeholders to the product design process and take into account the competitive environment, it is necessary to expand the product model and production processes to cover all the business processes of the enterprise, in fact, implementing the enterprise engineering approach. In this regard, the application of the Model-Based Enterprise (MBE) concept involves the introduction of advances in standards, testing methods and metrology which allow manufacturers to integrate models of systems, services, products, processes and logistics throughout the production enterprise [10]. At the same time, great importance is attached to ensuring that consumer qualities match the capabilities of their process implementation, as well as the best interoperability of decentralized distributed subsystems in order to form various product configuration versions for specific requirements specifications.

The MBE concept assumes the implementation of the following project principles [10]:

${ }^{1}$ Quality information framework: https://qifstandards.org/ 
$\downarrow$ integration of various types of static and dynamic models of intelligent production systems and analytical systems based on the interdisciplinary exchange of system and project information between them;

transformation of enterprises into service-oriented production systems that allow flexible building of business processes on a dynamic basis using modern digital technologies - IoT, AI, Big Data, etc.;

$\downarrow$ specification of a complex product structure using models, methods and tools that allow one to implement the concept of fully integrated production systems that are adaptive in real time to changing requirements and conditions within the enterprise and in the external environment;

$\downarrow$ visualization of product lifecycle data necessary for visual representation and perception of product information by participants of innovation and production processes;

identification of production capabilities that allow for evaluating the ability to meet the stated requirements for the product based on modeling the use of the provided resources at all stages of the life cycle;

synthesis and contextualization of information from traditionally incompatible sources to justify decisions in the operational management of product creation processes at different stages of the life cycle;

४ extracting and applying knowledge to perform operations based on hybrid intelligent systems in order to analyze unstructured information to ensure decision-making and continuous improvement of production systems.

The implementation of these principles is largely determined by the ability of hybrid intelligent knowledge-based systems to perform semantic mapping of the conceptual model of products and related processes in a multi-level ontology of the innovation pro- cess. In this regard, the work [11] on integrating models of created products within the framework of implementing a digital thread based on an ontological approach is known. The work is mainly devoted to the technological aspects of presenting various models in a single format that provides their semantic analysis. To do this, the SysML models of a certain product are converted to JSON documents and then converted to an RDF graph using the SysML ontology. In this case, the model dictionary can be automatically generated and compared with existing domain ontologies. If necessary, during sequential processing of RDF triplets, the identified new terms are added to the existing domain ontology. Then, as a result of a number of transformations and mapping to domain ontologies aligned using the BFO reference ontology, a refined RDF graph is formed, which is then used in the process of making innovative decisions. However, aspects related to multi-agent implementation of interaction between participants in the innovation process based on a multi-level system of ontologies and the corresponding cognitive interpretation of the interaction results are not disclosed in the article [11].

The proposed research focuses on the development of a functional architecture of a knowledge-based system that supports iterative dynamic decision-making on the configuration of new products and related processes with the participation of all stakeholders within the network enterprise. The main focus is on the initial stages of product creation, including the formation of requirements and design of the product and value chain, as a system of related production and logistics processes. At the same time, a multi-level system of ontologies of the innovation process is proposed as a system-forming component which provides semantic interoperability of knowledge about products and related processes. 


\section{Statement of the problem of developing a knowledge-based system for the design of innovative processes for creating products}

In accordance with the classical provisions of system engineering the process of designing products and services includes the following stages [2] (Figure 1):

Forming a product concept involves determining the consumer's value characteristics (business requirements) and evaluating them from the point of view of marketing attractiveness. In the future, the product concept is specified in terms of the possibility of technical implementation in the form of a set of functional and non-functional requirements.

Product construction design is the definition of a product structure based on an analysis of existing advances in the manufacturing of products and services, as well as promising technologies that can be used to create a product. The result of this stage is the component structure of the product and the definition of a list of technologies for the production of its components.
Detailed design of product creation processes involves the selection of network enterprise participants who will supply or produce certain product components in accordance with the specified requirements and technical design. Thus, at this stage, a value chain is formed that determines the distribution of work among the participants of the network enterprise. At this stage, a large amount of work is associated with coordinating the capabilities of suppliers and manufacturers to create certain components of products in accordance with design solutions and requirements.

Due to possible inconsistencies in the resource and other capabilities of the selected enterprises with the design solutions and requirements, iterative reversals are allowed during the product design process to review the project decisions and even changes in the product concept. This circumstance makes it necessary to dynamically maintain different versions of the project in order to choose the most effective solution version, taking into account all possible limitations (simultaneously maintaining a set of digital threads).

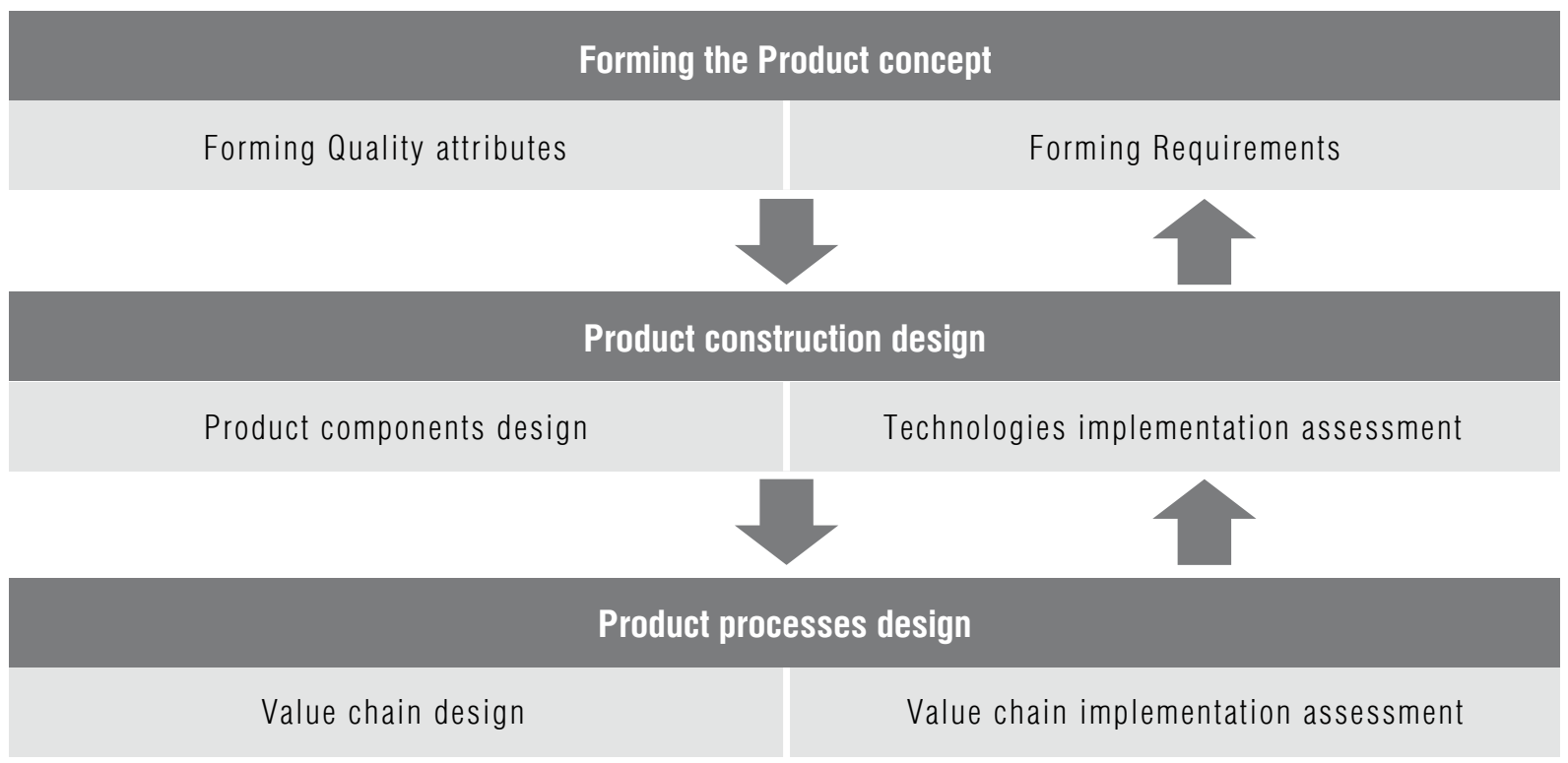

Fig. 1. Design stages of the innovative product creation process 
To justify the design solutions in existing works, it is proposed to use a modeling tool, which is selected depending on the nature of the problem being solved. For example, to solve marketing problems of justifying the choice of consumer qualities of products, these can be statistical models of market assessment, and to assess the performance of the designed product - simulation models. To integrate various types of models, it is proposed to use a system of project repositories united by a single object identification system that links all product-related artifacts at all stages of the lifecycle. The work [12] proposes the architecture for such a product lifecycle management system (System Lifecycle Handler, SLH), which is shown in Figure 2.

The main element of this system is a handle system that allows global identification for any artifact in the digital thread associated with the product project, as well as searching for related artifacts using a system of metadata descriptors such as (a) the artifact name, (b) the artifact type and schema, (c) the artifact author, (d) the artifact location, and (e) other attributes. The digital thread dashboard allows you to view, query, and visualize a digi- tal thread or any of its subgraphs at any stage of the lifecycle. The service system provides the following features:

$\checkmark$ connect to corporate data stores (repositories) such as PLM systems, alarm systems, requirements management systems, databases, and environment modeling tools;

$\checkmark$ search for individual artifacts;

$\downarrow$ linking artifacts from the various repositories and the implementation of the conversion from one form to another;

$\downarrow$ synchronization of changes in different repositories;

$\diamond$ graphical representation of the state of artifacts using various visualization tools.

Thus, the presented architecture of the SLH system basically implements the functions of an integrated knowledge management system, which allows maintaining consistent information about the process of creating and subsequent operation of the product. In contrast to the described product lifecycle management system, it is proposed to develop a knowledge-based system (KBS), which would allow, along with the specified components, to implement:

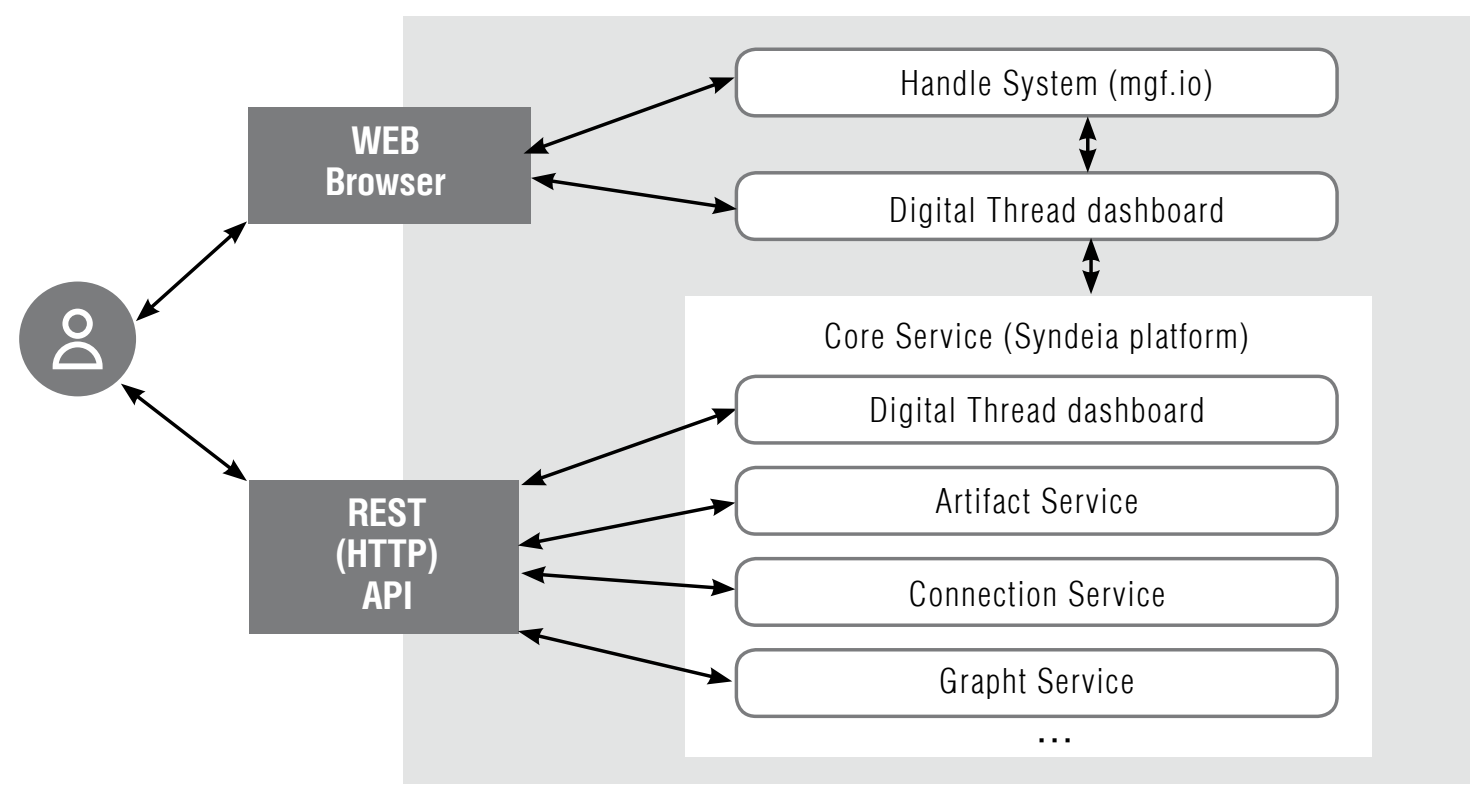

Fig. 2. Conceptual architecture of the product lifecycle management system [12] 
setting up mechanisms for solving the problem of building innovative processes on the specifics of the domain;

- integration of knowledge sources about products and related processes based on a single conceptual model of the domain;

assessment of the prospects for the application of design properties and requirements for products, components and technologies used;

design of value chains with the selection of participants in the innovation process with the best opportunities;

agreement of parameters of manufactured products with all interested participants of the innovation process.

In accordance with these requirements, a KBS architecture for designing innovative product creation processes is proposed (Figure 3). A central component is the ontology of the innovation process, which more deeply reflects the semantic conceptual model of the domain on the basis of which all the above requirements are implemented.
The main functional components correspond to the stages of the innovative product creation process. Each of them contains modules for selecting relevant artifacts from the corresponding data bases, ranking them by importance and evaluating them from the point of view of resource implementation capabilities. Project solutions are saved in the repository for different versions of the project from the point of view of the possibility of dynamically building and evaluating various versions for innovative processes and selecting the best version at each time.

Next, we will consider the features of implementing functional modules for designing innovative product creation processes from the point of view of forming design solutions based on the use of ontologies and databases of artifacts. Mechanisms for ranking design solutions based on the application of QFD and FMEA methods were considered earlier in [9], and the assessment of compliance of resource capabilities with value characteris-

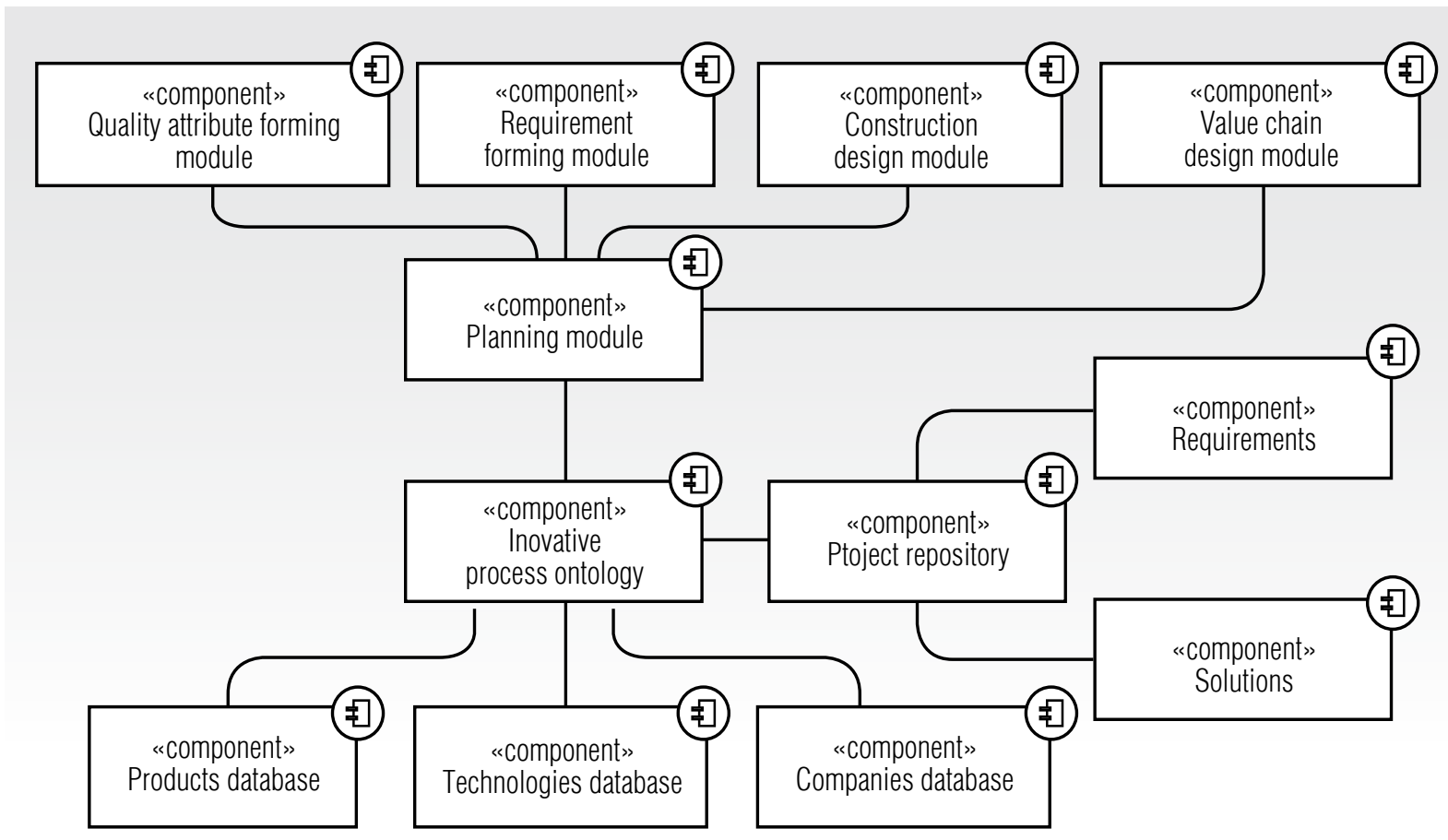

Fig. 3. The architecture of the KBS for the design of innovative processes for product development 
tics and functional requirements based on the application of fuzzy logic methods was considered in [13].

\section{Structure of the knowledge base for designing innovative processes for product creation}

In the architecture of KBS, a special place is occupied by the system of ontologies [14], which describes the structure of the innovative process of creating products in a network enterprise and the requirements for its organization, characteristic of various domains. This system provides integration of network enterprise databases into a single distributed knowledge base.

The following structure of ontologies is proposed, which will ensure the joint use of KBS by all participants in the innovation process of a network enterprise (Figure 4).

In particular, the system of ontologies includes the following elements:

metaontology or the description language used to describe all other ontologies; upper level reference ontolongy that contains key concepts, such as Object, Event, Space, Individual quality, and Time frame, and sets the approach to describing ontologies of domains. This ontology is used to align and share various lower level ontologies;

$\checkmark$ mid-level reference ontology related to the area of this research-organization of activities and production of network enterprises;

$\checkmark$ domain ontologies shared by participants in the value chain related to the production of a certain type of product that can be used by enterprises in the relevant industries and included in their knowledge base (refer to the reference ontology and contain product classifications, components, standard requirements and processes, etc.);

a applied ontology of the network enterprise, expanded from the upper and middle levels of ontologies, which is part of the knowledge base of the network enterprise and includes models of products and services produced, production chains and interaction patterns of the organization;

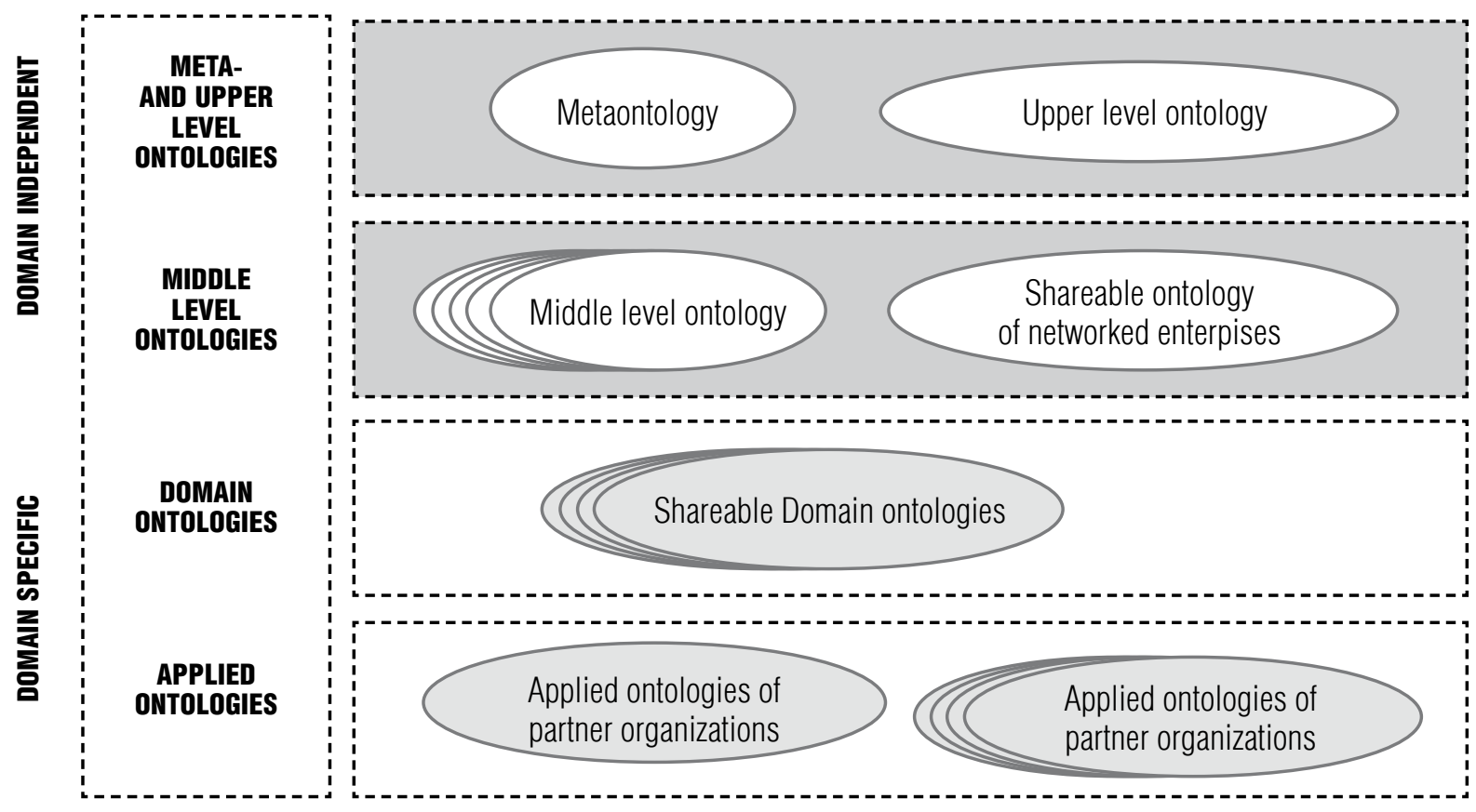

Fig. 4. Multilevel system of ontologies of KBS 
applied ontologies of participants in the value chain, extended from the upper and middle levels of ontologies, which are part of the knowledge bases of the relevant organizations and are used to describe the methods and practices used, information about the activities, products and contracts of the organization.

Heterogeneous data submitted by organizations can be integrated and converted into a standardized description format (in the form of an RDF graph) and aligned according to the ontologies used. Alignment is a prerequisite for making queries (in SPARQL or any other standardized language) to the unified knowledge base to control the integrity and implement the functions of the main modules.

The process of ontological alignment involves establishing correspondences between the concepts used in applied ontologies, and from the point of view of computer science is due to the need to integrate heterogeneous databases developed independently and using their own dictionaries. To facilitate this process, reference ontologies (top-level, middle-level, and subject-level) are used.

The language used for the formalization of ontologies determines metaontology. In the context of the Semantic Web project, or to ensure semantic interoperability of complex systems interacting over the Internet, OWL, a language developed by the World Wide Web consortium, is often used as such a language [15]. Languages based on first-order logic are also used for knowledge exchange in computer systems [16, 17].

When using a knowledge base represented as an RDF graph, OWL metaontology can be described based on the basic RDF schema
[18] and include built-in classes and properties that together form the basis of the RDF/ XML OWL 2 syntax. Decentralization of the network enterprise and the need to share knowledge bases of different organizations implies alignment and integration of applied ontologies. For these purposes, reference ontologies of the upper levels and reference ontologies of subject areas are used [19].

Among the well-known top-level reference ontologies, we can distinguish the BFO ontology, which is used by over 100 projects [20], GFO projects [21], DOLCE [22], and $\mathrm{SUMO}^{2}$. Among the reference subject ontologies of interest for the organization of the innovation process of a network enterprise are product ontologies, such as eClass OWL, GoodRelations and derivatives [23], Google taxonomy ${ }^{3}$, which are used to describe more than 30,000 types of products, nodes and components, as well as taxonomies of processes and activities [24].

For the organization of interaction and selection of participants of a network enterprise in a single information space, it is proposed to use the reference ontology of the innovation process, the key concepts of which are presented in the article [9].

An example of a system of related ontologies for designing innovation processes is shown in Figure 5. The resulting set of related data and ontologies is a distributed knowledge base of KBS. It can be used to ensure the interaction of organizations that are part of the network enterprise structure at various levels - both for describing quality characteristics, and when designing a production system, organizing the value chain, and the innovation process.

\footnotetext{
2 Suggested Upper Merged Ontology (SUMO): http://www.ontologyportal.org/

${ }^{3}$ Google Product Taxonomy: https://www.google.com/basepages/producttype/taxonomy.en-US.txt
} 

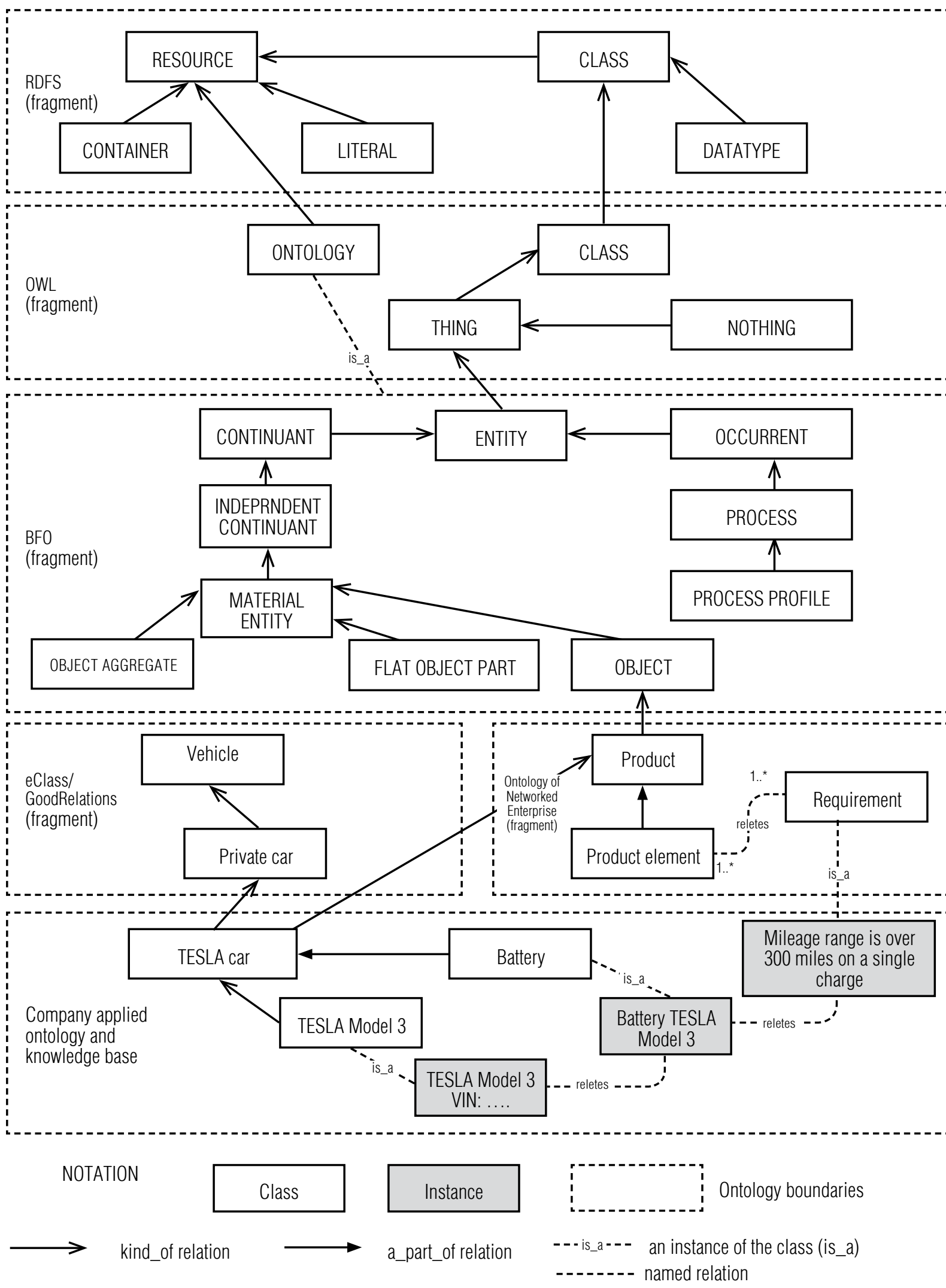

Fig. 5. Example of a related ontologies system for designing innovation processes 


\section{Organization of interaction between participants in the value chain of a network enterprise}

The use of a distributed knowledge base in the process of design a value chain and the structure of a network enterprise is carried out within the framework of five main modules:

$\downarrow$ planning module (coordinator);

$\downarrow$ quality attribute forming module;

$\checkmark$ requirements forming module;

$\downarrow$ construction design module;

$\downarrow$ value chain design module.

The planning module receives information about the type of Product being designed, determines the order and calls the corresponding modules, analyzes the results obtained, and initiates clarification of the missing information. To justify the choice of quality char- acteristics and product requirements, as well as product construction versions and value chains, an approach based on a combination of QFD and fuzzy logic methods is used $[9,25]$.

The quality attribute forming module is used at the stage of forming a Product concept to identify and formalize Quality attributes (Figure 6) through which the value of the Product for the consumer is determined.

The reference ontology defines the general concepts of Product and Attribute, as well as the types of attributes in accordance with the Kano method [26]. In shared ontologies, Product types that are specific to a particular domain are added. The organization's knowledge base stores descriptions of different types of typical attributes for different types of products. This knowledge is accumulated in the course of research on consumer preferences and can be used to determine the attributes of a new product, so that:

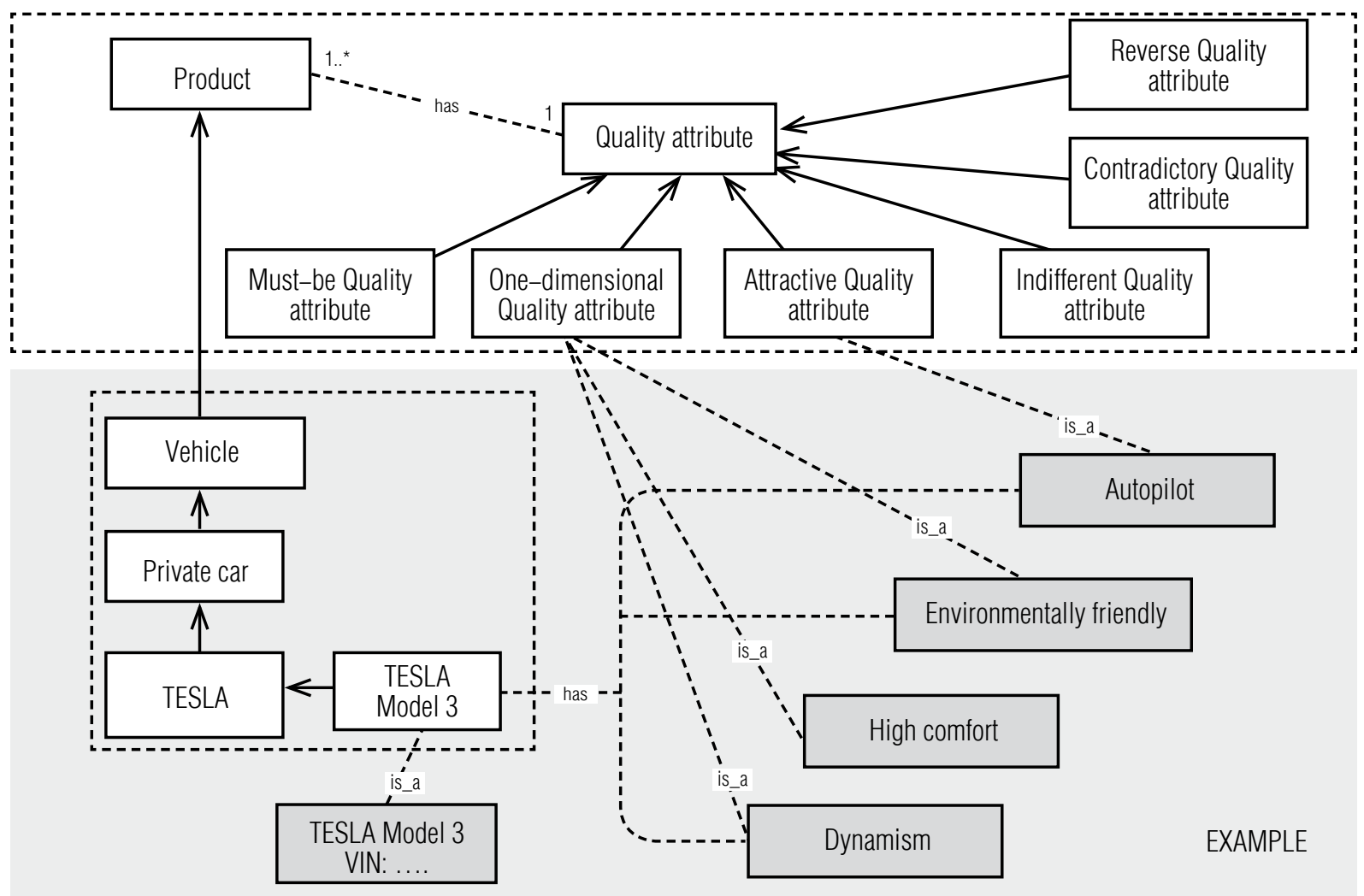

Fig. 6. A fragment of the reference ontology: an example of describing the quality attributes of a product 
\ selects the Product type corresponding to the specified one;

$\triangleleft$ a set of Product attributes of different types is generated from the knowledge base that is specific to this type of Product;

$\checkmark$ all Required attributes will be associated with the new Product;

$\checkmark$ the Expected and Attractive attributes are evaluated and included in the set;

$\diamond$ minimizes the presence of Non-Influencing, Contradictory and Distasteful attributes.

The received attributes are returned to the planning module, selected, and passed on to form a set of requirements.

For each mandatory or expected characteristic, at least one related requirement must be formulated. The formalization of requirements is made using the requirements forming module. Within this module, qualitative Attributes are specified in terms of functional and nonfunctional Requirements for the Product that ensure the implementation of these Attributes [27] (Figure 7).

The top-level ontology includes a description of the concept of a Requirement and its relationships with other concepts (for example, an Attribute, Function, or Product element). Organizations' knowledge bases can store Standard requirements specific to certain Product categories.

When creating new Product requirements, the Original requirements are generated. Based on the Standard requirements, an Original requirement is usually created that refers to the Standard requirements (for example, "take into account the requirements of GOST R ISO 12003"). Some Implied requirements may be highlighted based on previous experience from the organization's knowledge base.

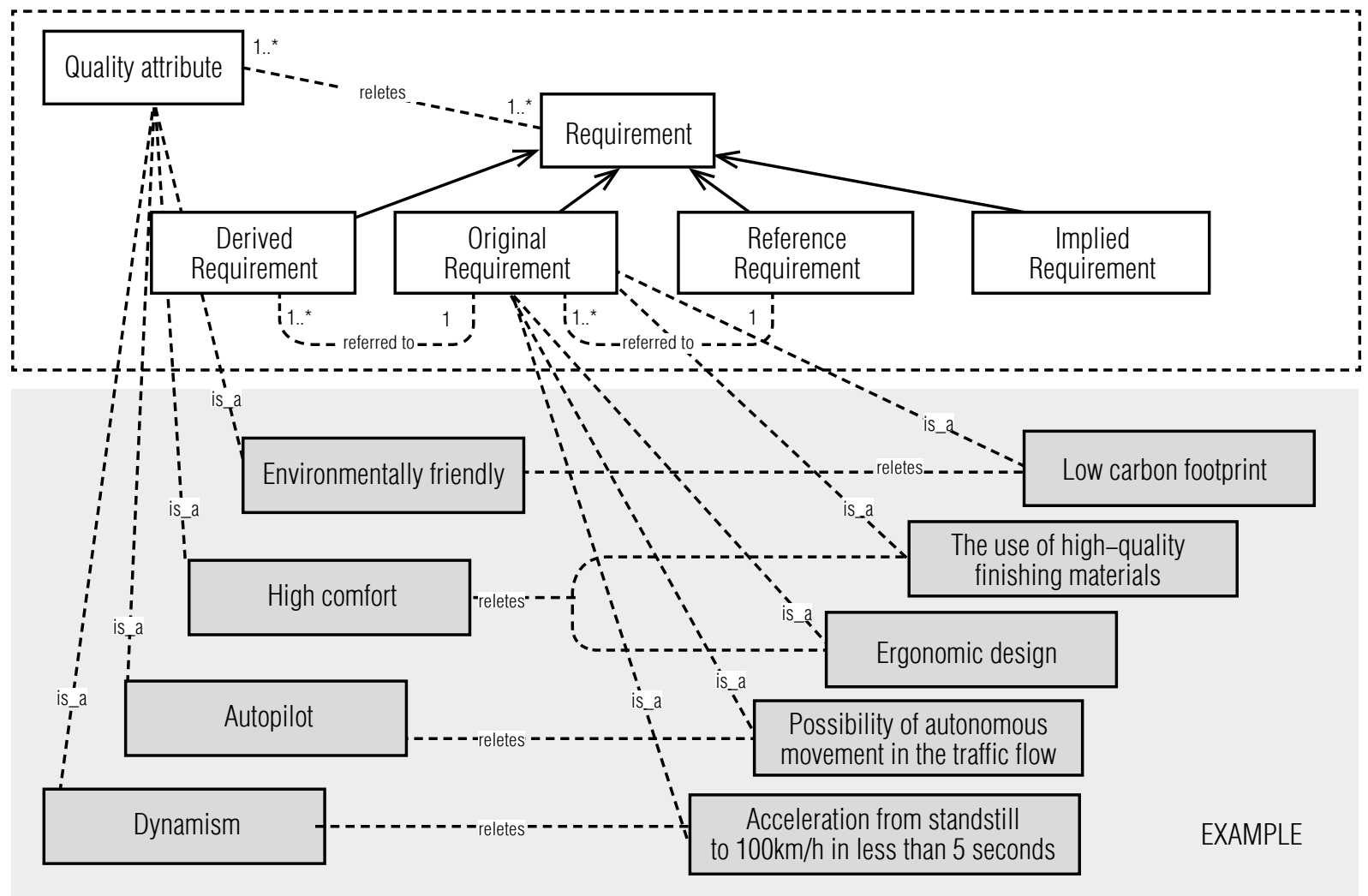

Fig. 7. A fragment of the reference ontology. An example of the description of the product requirements 
To conduct further analysis, it is necessary to clarify the wording of requirements as much as possible, including by separating complex Original requirements and separating Derived requirements from their composition.

The resulting set of requirements is returned to the planning module. Fuzzy estimation is used to determine the characteristics of requirements. The priority of a characteristic depends on its type: for required, expected and attractive characteristics it is equal to one, for adverse ones is equal to zero, for non-binding and contradictory characteristics - a fuzzy scale of the certainty coefficient $[0 ; 1]$ is applied.

As a result of evaluating the resulting set of requirements, the planning module calls the next module or refers to the previous one for clarification.

Within the framework of the construction design module, the component composition is formalized, the structural Elements of the product and its Functions are highlighted.

A requirement may be related to some Element or Function of a product, either explicitly or indirectly, through the choice of production method, technology, sources of information/ knowledge used, regulations and tools. In this way, the design is defined through the Requirements (Figure 8).

Constructive Elements can be included in the Product structure to provide certain functions, mandatory interface requirements, or as a result of inheriting from a typical Product construction that has a product breakdown structure (PBS) that references product ontologies available in the knowledge base.

Based on typical solutions, a product variant (version) or Product project is formed, which combines many types of structural Elements and Functions performed by the product.

Each Project is reflected in structural Models (the composition of structural elements, components or parts, structural and full-scale) and functional Models (product scenarios, simula- tion models), which confirm compliance with the initial Requirements.

The Product project (project version) is selected by the planning module using a combination of QFD and fuzzy logic methods. Evaluating the total importance of Elements included in the Product construction allows you to rank versions. But the determining factor is whether the selected types of structural elements can be purchased and/or whether they can be produced in accordance with existing Models.

To evaluate this possibility, the planning module calls the value chain design module, which is used to analyze the market and select participants in the innovation Process that has several Versions. In order to choose the Version of Prod$u c t$ process, it is necessary to organize interaction between different Enterprises based on their competencies, formalized and published in open sources (Figure 9). The process of matching the requirements and capabilities of enterprises is carried out using multi-agent technology [28].

Providers of structural elements or raw materials that are represented in some version of the Product construction are searched in the register of Enterprises. For each Provider, the ability to deliver a certain category of Elements is evaluated. As initial requirements, the Provider receives a description of the Element, formalized through links to the product ontology and existing structural and functional Models that describe the Element and its purpose, as well as design Requirements.

The absence of constructive Element from Providers associated with mandatory Requirements can lead to one of the following organizational scenarios initiated by the planning module:

$\checkmark$ completion of work due to the lack of product Elements necessary for creating the Product;

$\checkmark$ revision of the Product concept in order to clarify the characteristics and requirements;

$\downarrow$ selecting a different Product construction version according to the rank; 


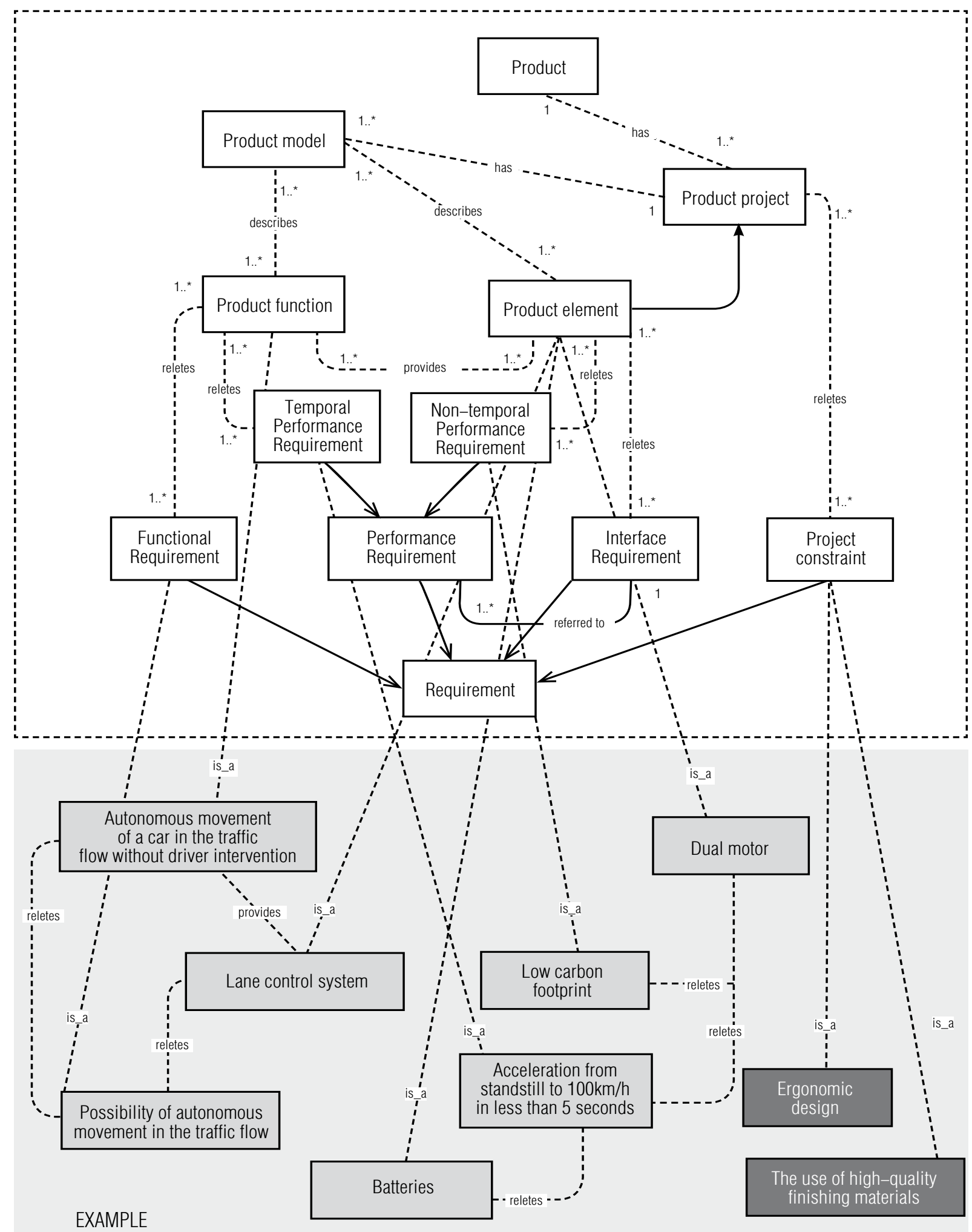

Fig. 8. A fragment of the reference ontology: an example of a product project description 


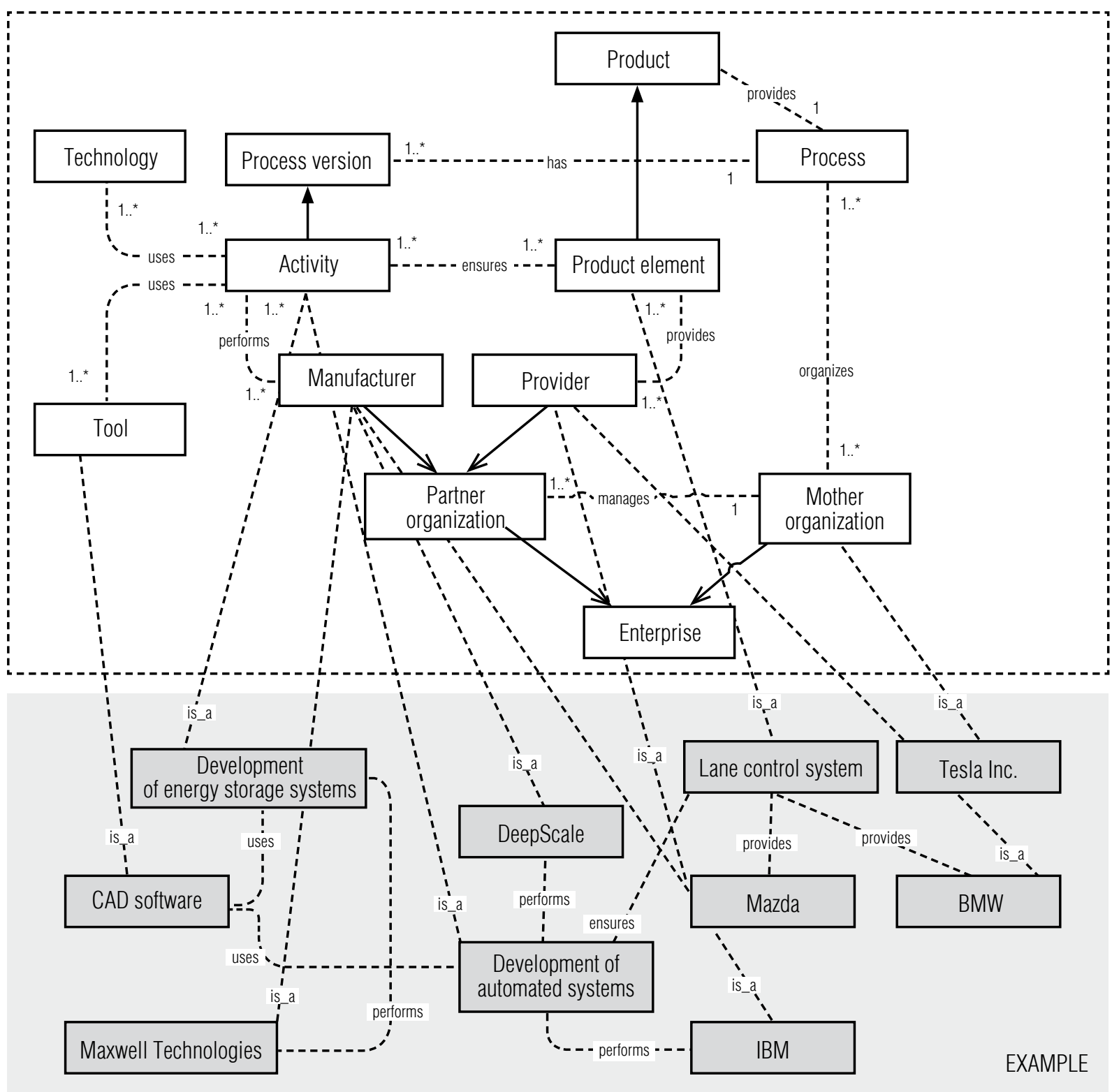

Fig. 9. A fragment of the top-level reference ontology: an example of a description of participants in the value chain

select the version of producing the Element to order using the necessary technologies and tools.

In order to identify a Manufacturer that can produce custom Elements according to the Product construction, Process versions are formed. These enterprises could perform work on the production of Elements, as well as work on the design, assembly, processing, adjustment, main- tenance and delivery of products. The composition and relationships of Activities are contained in the domain ontology. For each of these types of Activities, possible Tools and Technologies are identified that ensure the production of constructive Elements with specified Design constraints and that meet the established Requirements.

The Process versions are evaluated by the planning module in the same way that Product pro- 
jects are evaluated using QFD and fuzzy logic methods. Evaluating the total importance of all Activities using the QFD method allows you to rank versions in the process chain and select Manufacturers based on their competence requirements (ability to perform Activities, knowledge of Tools and Technologies). The algorithm for evaluating deviations of Requirements from the capabilities of the Enterprise based on fuzzy logic is considered in the article [13], while determining the possibility of performing Activities on their own or outsourcing it.

The search for Manufacturers is carried out by comparing the description of the type of Activity and the corresponding Tools and Technologies with the facts presented in the databases of enterprises. If there are no Enterprises that fully meet the specified conditions, then the process is switched to other versions according to the previously defined rank.

A process version with a list of Activities that must be performed to form the final Product construction is saved by the planning module in the knowledge base of the network enterprise and used at the production stage for coordinating work, as well as for developing new types of products.

\section{Conclusion}

As a result of the study of methods for designing innovative processes for creating products of a network enterprise and their implementation in knowledge-based systems, the following conclusions can be drawn:

$\downarrow$ within the framework of the innovation process, the most preferable approach is to build enterprises based on models (Model Based Enterprise, MBE), which best implements the concept of "digital thread";

$\downarrow$ to organize the "digital thread" within the framework of the proposed knowledge-based system, it is necessary to use a distributed knowledge base and a multi-level ontology system for designing innovative processes of prod- uct creation which displays both the typical structures of products and value chains, as well as the accumulated production experience and capabilities of enterprises;

$\downarrow$ to organize the functioning of KBS in the structure of the system there should be allocated modules for the formation of quality characteristics of the product, requirements for the product, the product construction and value chains (the process of its production), as well as the planning module;

$\downarrow$ within the framework of designing the structure of the innovation process of a network enterprise, it is advisable to use a combination of various methods (QFD and fuzzy logic) that ensure the best choice of product construction, value chains and the composition of enterprises participating in the value chain.

The novelty of the proposed knowledge-based system architecture for the design of an innovation process lies in the possible iteration of this process, which allows us to find the best design solutions for both the product construction and the value chain. In addition, the implementation of this architecture will improve the semantic interoperability of participants in the innovation process, and, consequently, the quality of innovative products created.

As part of the further development of the proposed methods and approaches to the development of KBS for the organization of the innovative process of product creation, it is advisable to develop algorithms for individual modules of the system, methods and tools for integrating ontologies included in the distributed knowledge base, methods of multi-agent interaction of network enterprise participants that allow one to dynamically adapt the structure of the innovation process of a network enterprise to requirements that change at all stages of the product lifecycle.

\section{Acknowledgments}

This work was supported by the RFBR, grants no 19-07-01137a, 18-07-01053a, 18-07-00918a. 


\section{References}

1. Namestnikov A.M. (2009) Smart project repositories. Ulyanovsk: USTU (in Russian).

2. Baker L., Clemente P., Cohen R., Permenter L., Purves B., Salmon P. (2000) Foundational concepts for model driven system design. White paper. INCOSE Model Driven System Design Interest Group, International Council on Systems Engineering.

3. Frechette S.P. (2010) Model based enterprise for manufacturing. Manufacturing Systems Integration Division, Engineering Laboratory, National Institute of Standards and Technology.

4. Minaev V.A., Mazin A.V., Zdiruk K.B., Kulikov L.S. (2019) Digital twins of objects in the solution of control problems. Radio Industry (Russia), vol. 29, no 3, pp. 68-78 (in Russian). DOI: $10.21778 / 2413-9599-2019-29-3-68-78$

5. United States Air Force (2013) Global Horizons. Final Report: United States Air Force Global Science and Technology Vision - AF/ST TR 13-01. 21 June 2013. Available at: https://www.airforcemag.com/ PDF/DocumentFile/Documents/2013/GlobalHorizons_062313.pdf (accessed 01 March 2020).

6. NIST (2020) Digital thread for smart manufacturing. Available at: https://www.nist.gov/programsprojects/digital-thread-smart-manufacturing (accessed 20 June 2020).

7. Uschold M., King M., Moralee S., Zorgios Y. (1998) The enterprise ontology. The Knowledge Engineer Review, vol. 13, no 1, pp. 31-89. DOI: 10.1017/S0269888998001088.

8. Dietz J.L.G. (2006) Enterprise ontology: Theory and methodology. Berlin, Heidelberg: Springer-Verlag. DOI: $10.1007 / 3-540-33149-2$.

9. Telnov Yu.F., Trembach V.M., Danilov A.V., Yaroshenko E.V., Kazakov V.A., Kozlova O.A. (2019) Constructing network enterprise structure to create innovative products. Open Education, vol. 23, no 6, pp. 59-73 (in Russian). DOI: 10.21686/1818-4243-2019-6-59-73.

10. NIST (2020) Model-based enterprise program. Available at: https://www.nist.gov/programs-projects/ model-based-enterprise-program (accessed 20 June 2020).

11. Bone M., Blackburn M., Kruse B., Dzielski J., Hagedorn T., Grosse I. (2018) Toward an interoperability and integration framework to enable digital thread. Systems, vol. 46, no 6. Available at: https://www.mdpi.com/2079-8954/6/4/46 (accessed 01 March 2020). DOI: 10.3390/systems6040046.

12. Bajaj M., Hedberg T. (2018) System lifecycle handler - Spinning a digital thread for manufacturing. Proceedings of the 28th Annual INCOSE International Symposium, Washington, DC, 7-12 July 2018, pp. 1626-1650. DOI: 10.1002/j.2334-5837.2018.00573.x.

13. Kalachihin P.A., Telnov Yu.F. (2018) Formation of value chains in network interaction structures based on intelligent technologies. Proceedings of the XVI National Scientific Conference on Artificial Intelligence with International Participation, Moscow, Russia, 24-27 September 2018, vol. 1, pp. 106-115 (in Russian).

14. Rudnicki R., Smith B., Malyuta T., Mandrick W. (2016) Best practices of ontology development. White paper. Available at: https://www.nist.gov/system/files/documents/2019/05/30/nist-ai-rfi-cubrc_inc_002. pdf (accessed 01 March 2020).

15. W3C (2012) OWL 2 Web Ontology Language document overview (Second edition). Available at: http://www.w3.org/TR/owl2-overview/ (accessed 01 March 2020).

16. Pease A. (2009) Standard Upper Ontology Knowledge Interchange Format (SUO-KIF). Available at: https://github.com/ontologyportal/sigmakee/blob/master/suo-kif.pdf (accessed 01 March 2020).

17. ISO (2018) ISO/IEC 24707:2018. Information technology - Common Logic (CL) - A framework for a family of logic-based languages. Available at: https://www.iso.org/standard/66249.html (accessed 01 March 2020).

18. W3C (2014) The RDF Schema vocabulary (RDFS). Available at: https://www.w3.org/TR/rdf-schema/ (accessed 01 March 2020).

19. Brinkley J.F., Suciu D., Detwiler L.T., Gennari J.H., Rosse C. (2006) A framework for using reference ontologies as a foundation for the semantic web. AMIA Annual Symposium Proceedings, pp. 96-100. Available at: https://www.ncbi.nlm.nih.gov/pmc/articles/PMC1839690/ (accessed 01 March 2020). 
20. Smith B. (2015) Basic Formal Ontology 2.0. Specification and user's guide. Available at: https://raw.githubusercontent.com/BFO-ontology/BFO/v2.0/BFO2-Reference.docx (accessed 01 March 2020).

21. Herre H., Heller B. Burek P., Loebe F., Hoehndorf R., Michalek H. (2006) General Formal Ontology (GFO) - A foundational ontology integrating objects and processes. Report Nr. 8. OntoMed, IMISE.

22. Borgo S., Masolo C. (2010) Ontological foundations of DOLCE. Theory and applications of ontology: Computer applications (R. Poli, M. Healy, A. Kameas, eds). Dordrecht: Springer.

DOI: 10.1007/978-90-481-8847-5_13.

23. Hepp M., Radinger A. (2010) eClass OWL - The web ontology for products and services. Available at: http://www.heppnetz.de/projects/eclassowl/ (accessed 01 March 2020).

24. APQC (2016). Cross-industry process classification framework. Available at: https://www.apqc.org/ system/files/K08175_Cross_Industry_v7.0.5_russian.pdf (accessed 01 March 2020) (in Russian).

25. Vashchukov Yu.A., Dmitriev A.Ya., Mitroshkina T.A. (2012) QFD: Product and process development based on customer requirements and expectations. Samara: SSAU (in Russian).

26. Kano N., Seraku N., Takahashi F., Tsuji S. (1984) Attractive quality and must-be quality. Journal of Japanese Society for Quality Control, vol. 14, no 2, pp. 39-48.

27. Oliver D.W., Kelliher T.P., Keegan J.G., Jr. (1997) Engineering complex systems with models and objects. McGraw-Hill.

28. Telnov Yu.F., Danilov A.V., Diveev R.I., Kazakov V.A., Yaroshenko E.V. (2018) Development of a prototype of multi-agent system of network interaction of educational institutions. Open Education, vol. 22, no 6, pp. 14-26 (in Russian).

\section{About the authors}

\section{Yury F. Telnov}

Dr. Sci. (Economics), Professor;

Head of the Department of Applied Information Technologies and Information Security,

Plekhanov Russian University of Economics,

36, Stremyanny Lane, Moscow 117997, Russia;

E-mail: Telnov.YUF@rea.ru

ORCID: 0000-0002-2983-8232

\section{Vasiliy A. Kazakov}

Cand. Sci. (Economics);

Leading Researcher, Research Institute "Strategic Information Technologies",

Plekhanov Russian University of Economics,

36, Stremyanny Lane, Moscow 117997, Russia;

E-mail: Kazakov.VA@rea.ru

ORCID: 0000-0001-8939-2087

\section{Vasiliy M. Trembach}

Cand. Sci. (Tech.), Associate Professor;

Associate Professor, Department 304, Moscow Aviation Institute (National Research University), 4, Volokolamskoe Shosse, Moscow 125993, Russia;

E-mail: trembach@yandex.ru

ORCID: 0000-0001-7499-4368 